\title{
Localized Castleman Disease
}

National Cancer Institute

\section{Source}

National Cancer Institute. Localized Castleman Disease. NCI Thesaurus. Code C115200.

Angiofollicular lymphoid hyperplasia that presents with localized lymphadenopathy. 